\title{
OPTIMUM TOPOLOGICAL CONFIGURATIONS OF SENSOR NETWORKS
}

\author{
Rita Greco ${ }^{1}$, Giuseppe Carlo Marano ${ }^{2}$ \\ ${ }^{1}$ DICATECH, Department of Civil Engineering, Environmental, Territory, Building and Chemical, \\ Polytechnic of Bari -Italy. \\ ${ }^{2}$ DISEG, Department of Structural, Building and Geotechnical Engineering, \\ Polytechnic of Turin - Italy
}

\begin{abstract}
Placing sensors at appropriate locations is an important task in the dynamic assessment of the mechanical systems and its role is central in structural health monitoring. All sensors have to be placed on the structure so that all sought system features be obtained from the experimental tests. Sensors network design is rather diffused in laboratory applications, but there are scarce investigations on real civil structures and they are not so well documented. In order to give a contribution in this framework, the present paper deals with the optimal sensors placement problem on lattice towers. With reference to six of the most diffused existing optimum criteria and according to mechanical and energetic formulations, several sensors network configurations are calculated for two example broadcasting antennas, adopted as benchmark cases of studies. The final topological configurations of the networks are compared and the most relevant differences are discussed. Moreover, the information content behaviour is computed to investigate the connection between sensors network topology and its ability to withstand the presence of undesired signals (e.g., noise) during the measurements. The results here illustrated may be useful for sensors network design for this special class of structures.
\end{abstract}

\section{Introduction}

The dynamic assessment of real mechanical systems is possible only if a minimum amount of information (the structural response under natural or artificial dynamic loads) is acquired. In this aim, the investigated system must be equipped with a minimum number of sensors. However, this is not the only "constraint" of the problem: others, of economic and technological nature, impose the installation, of a maximum number of sensors, too. In order to monitor the most significant information it is all too evident that sensors must be accurately positioned. In this sense, from a mathematical standpoint, the optimal sensor placement (OSP) is a constrained topologic combinatorial (or mixed) optimization problem. As for any optimization problem, the optimum criterion (the objective function of the optimization problem) should be defined for the goal of the sensors positioning and should be selected with reference to the investigated mechanical system. Thus, its role is fundamental and it is not surprising that great efforts have been exerted in the last two decades to define suitable formulations.

One of the most used approaches is the so-called Effective Independence Method (EIM) presented in (Kammer and Brillhart 1996) and developed for inorbit modal identification of large space structures. This method uses the determinant of the Fisher information matrix to define the optimal sensors array. In order to maximize the measured kinetic energy of the mechanical system, a modified version of the EIM, the Kinetic Energy Method (KEM), has been developed in (Wang et al. 1998): in this case, the original Fisher information matrix is weighted with the mass matrix to compute the energy matrix.

Different from the methods based on the Fisher information matrix, in (Papadimitriou 2004) an entropy-based concept is developed to formulate an optimum criterion for the OSP problem: the Author uses the entropy measure (a decreasing function of the total sensor number) to characterize the amount of useful information contained in the available set of measured data. Alternative methods for sensors and actuators placement are based on controllability and observability measures, see for instance (Gawronski et al. 1997) or (Shih et al. 1998). Damage-based optimum criteria can be formulated for the OSP problem, as well: in this case, the goal is to find an optimal configuration of sensors that will best predict structural damage. For instance, in (Shi et al. 2000) sensors are positioned according to their damage localization ability evaluated by means of the eigenvector sensitivity method.

For the sake of completeness, it is necessary to remark that existing approaches differ also for the solution strategies. This is not a marginal question in the OSP, especially if a large number of candidate positions have to be examined. A very competitive technique is the sequential sensors placement: in this case, degreesof-freedom (DOFs) to equip with a sensor are selected one at a time, according to the adopted optimum criterion. This strategy can be applied in several methods, either based on the Fisher information matrix or on the entropy measure. An alternative way can be proposed by adopting a global search in solving the OSP problem. For instance, the use of genetic 
algorithms for the OSP is proposed in (Guo et al. 2004) and (Liu et al. 2008). Artificial neural networks and other non-classical combinatorial optimizers are used in the OSP for fault detection in (Worden and Burrows 2001), while the "ant colony algorithm" is performed in (Overton and Worden 2004).

Generally speaking, due to the existence of a wide number of optimum criteria, it is advisable to produce several sensors arrays using different formulations and to compare the obtained configurations according to some performance indicators. For example, (Meo and Zumpano 2005) analyze the difference between several criteria for the optimal sensors positioning on the Nottingham bridge. In this paper, the comparison between six different optimum criteria has been performed with reference to two example broadcasting antennas. The final topological configurations are presented and the principal differences are discussed. Robustness of the sensors arrays against undesired signals (e.g., noise) during the measurements is evaluated. Results here illustrated may be useful for sensors network design of this special structures.

\section{Optimum Criteria For Sensors Placement}

In this Section, we briefly illustrate the adopted criteria for the OSP in deterministic mechanical systems with linear behaviour.

\section{Effective Independence Method}

The EIM deals with the goal to maximize both the spatial independence and signal strength of the target mode shapes by maximizing the determinant of the associated Fisher information matrix. For a linear system, in modal space the structural response $\mathbf{y}$ can be expressed as

$$
\mathbf{y}=\boldsymbol{\Phi q}+\mathbf{w}
$$

where $\boldsymbol{\Phi}$ is the matrix collecting $N$ target mode shapes, $\mathbf{q}$ is the coefficient response vector and $\mathbf{w}$ the zero mean White Gaussian Noise (WGN) vector

$$
\mathbf{w} \sim \mathrm{N}(\mathbf{0}, \sigma \mathbf{I})
$$

and $\mathbf{I}$ is the identity matrix. In (2), $\sigma$ is the standard deviation of the random noise (without loss of generality, it is assumed equal for any measurement point).

The linear assumption is generally fulfilled in usual dynamic testing especially if one is interested to monitor the system response under small or moderate dynamic loads. By evaluating the coefficient response vector $\mathbf{q}$ through an efficient unbiased estimator, it is possible to estimate the covariance matrix as follows

$$
\mathbf{C}_{\hat{\mathbf{q}}}=\mathrm{E}\left[(\mathbf{q}-\hat{\mathbf{q}})(\mathbf{q}-\hat{\mathbf{q}})^{T}\right]=\sigma^{2}\left(\boldsymbol{\Phi}^{T} \boldsymbol{\Phi}\right)^{-1}
$$

that results the inverse of the Fisher information matrix, according to the Cramer-Rao Lower Bound (CRLB) theorem $(\mathrm{E}[\cdot]$ denotes the expected value operator). In virtue of the fact that the best estimation of $\mathbf{q}$ occurs when the Fisher information matrix is maximized, the procedure for selecting the OSP is to unselect candidate sensor positions such that its particular crisp value (trace or determinant) is maximized.

In an iterative manner, from a computational point of view, the analysis starts by computing the effective independence distribution (EID) vector

$$
\boldsymbol{\varepsilon}=[\boldsymbol{\Phi} \boldsymbol{\Psi}] \times[\boldsymbol{\Phi} \boldsymbol{\Psi}] \boldsymbol{\Lambda}^{-1} \mathbf{1}
$$

In (4), $\boldsymbol{\Psi}$ is the eigenvector matrix of $\boldsymbol{\Phi}^{T} \boldsymbol{\Phi}$ and $\boldsymbol{\Lambda}$ the associated eigenvalue matrix, symbol $\times$ denotes the Hadamard product (term-by-term matrix multiplication), $\mathbf{1}$ is the $N \times 1$ vector with all elements equal to one. Operatively, the selection procedure sorts the element of $\varepsilon$ and removes the smallest one in each iteration. Thus, the effective independence distribution vector (4) is updated, according to the new modal shapes matrix. The process is restarted until the number of remained sensors equals a desired value.

\section{Driving point residue method}

The modal participation factor or Driving Point Residue (DPR) is a mechanic optimum criteria (Doebling 1995). The DPR for the $i$-th DOF can be evaluated by using this expression

$$
\mathrm{DPR}_{i}=\sum_{j=1}^{N} \frac{\varphi_{i j}{ }^{2}}{\omega_{j}}
$$

where $\omega_{j}$ is the $j$-th target mode frequency ( $\varphi_{i j}$ is the $i$ th nodal displacement of the $j$-th mode shape). In this case, sensors will be placed where the DOF is characterized by the highest average response over all of the target modes. This method has been adopted by (Meo \& Zumpano 2005) coupled with the EIM strategy (EIM-DPR method). In fact, by multiplying the candidate sensor contribution of the EIM with the corresponding DPR coefficient in (5), it is possible to avoid candidate positions with low energy content.

\section{Kinetic energy based method}

In KEM the objective is to maximize the measure of the kinetic energy of the structure. In this case, the Fisher information matrix is weighted with the mass matrix $\mathbf{M}$ and so, the kinetic energy matrix $\mathbf{V}$ can be defined as follows

$$
\mathbf{V}=\boldsymbol{\Phi}^{T} \mathbf{M} \boldsymbol{\Phi}
$$

Decomposing the mass matrix into lower (denoted $\mathbf{L}$ ) and upper (denoted $\mathbf{U}$ ) triangular Cholesky factor matrices 


$$
\boldsymbol{\Gamma}=\mathbf{U} \boldsymbol{\Phi} \quad \mathbf{M}=\mathbf{L} \mathbf{U}
$$

the kinetic energy matrix can be defined as the product of the matrix $\Gamma$ and its transpose

$$
\mathbf{V}=\boldsymbol{\Gamma}^{T} \boldsymbol{\Gamma}
$$

After the evaluation of $\boldsymbol{\Gamma}$, the OSP process is identical to that described for the EIM.

\section{Eigenvalue vector product method}

Eigenvalue Vector Product (EVP) is a mechanic type criteria (Doebling 1995). This criteria forms an indicator consisting in the absolute value of the product of every eigenvector. This product can be written for the $i$-th structural DOF over the measured modes as

$$
\mathrm{EVP}_{i}=\prod_{j=1}^{N}\left|\varphi_{i j}\right|
$$

Following this criteria, the best locations are those with the highest EVP. The main advantage of this criteria is that it is able to prevent the location of sensors (but also of actuators) on nodal lines of a vibration modes, so that the maximization of their vibration energy is expected.

\section{Non-optimal driving point method}

It is an energy-based method, generally used to find the optimal location for actuators. Non-optimal driving point based method (NODP) is based on the well-known concept that the amount of vibration energy of any mode shapes depends on the relative positions of the excitation sources.

Following this way, the proposed methodology looks for the OSP by adopting an iterative procedure that unselects the candidate sensor having the smallest target mode shape displacement, that is:

$$
\mathrm{NODP}_{i}=\min _{j}\left|\varphi_{i j}\right|
$$

\section{Numerical Applications}

A wide class of engineering applications deals with the use of metallic lattice towers. They can be adopted in telecommunications, radio and television broadcasting, observation, power transmission and lighting supports, just to name some of the most diffused uses.

Different from other kind of engineering structures, the occurrence of the failure event generally does not involve the loss of human life, unless the tower is placed in a densely populated area. On the other hand, it is well known that also moderate dynamic loads can lead to temporary but frequent malfunctions, for instance, due to the loss of pointing accuracy in the installed equipment or devices. Moreover, the presence of acceleration-sensitive instruments could require the reduction of the detrimental inertial forces induced by vibrations. In order to measure the structural response, an efficient OSP is required because the off-line conditions in these structures is often economically relevant.

Under this auspicious, EIM, EVP, NODP, DPR, EIMDPR and KEM will be used to define appropriate topological network configurations for two example broadcasting antennas. All analyses for resolving the OSP problems are carried out by means of SAP2000 (version 11).

\section{Tower A}

The finite element 2D model and ten modes for Tower A are depicted in Figure 1. The FE model has 146 joints and two nodes connected to the base (total number of DOF is 288). It is constituted of 16 different type frames with area in the range $430 \mathrm{~mm}^{2} \div 3000$ $\mathrm{mm}^{2}$.

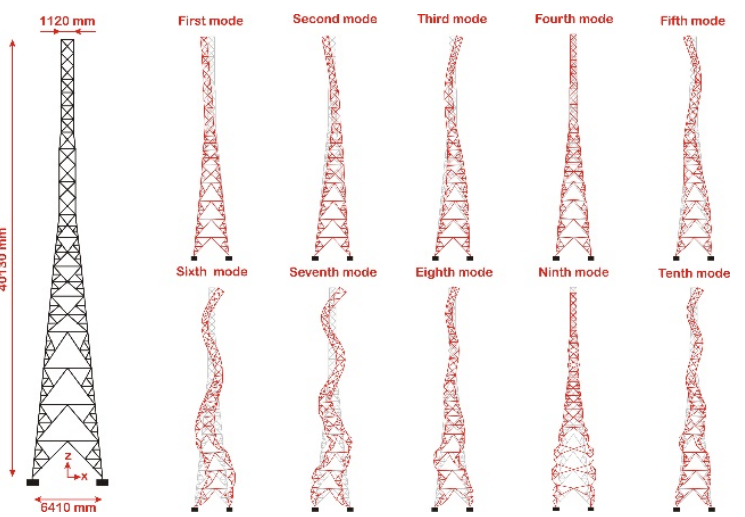

Figure 1. Finite element model for Tower A and first ten mode shapes

By using ten modes and the above listed optimum criteria, we obtain the configurations indicated in Figure 2.

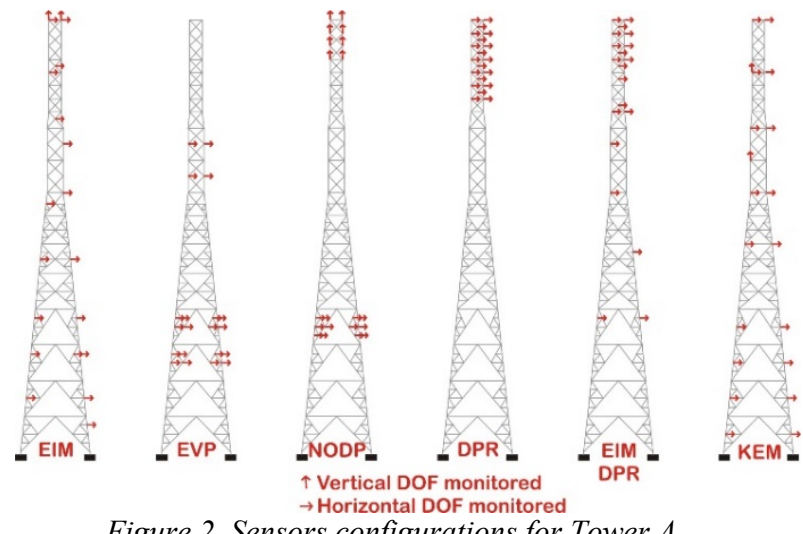

Figure 2. Sensors configurations for Tower A 


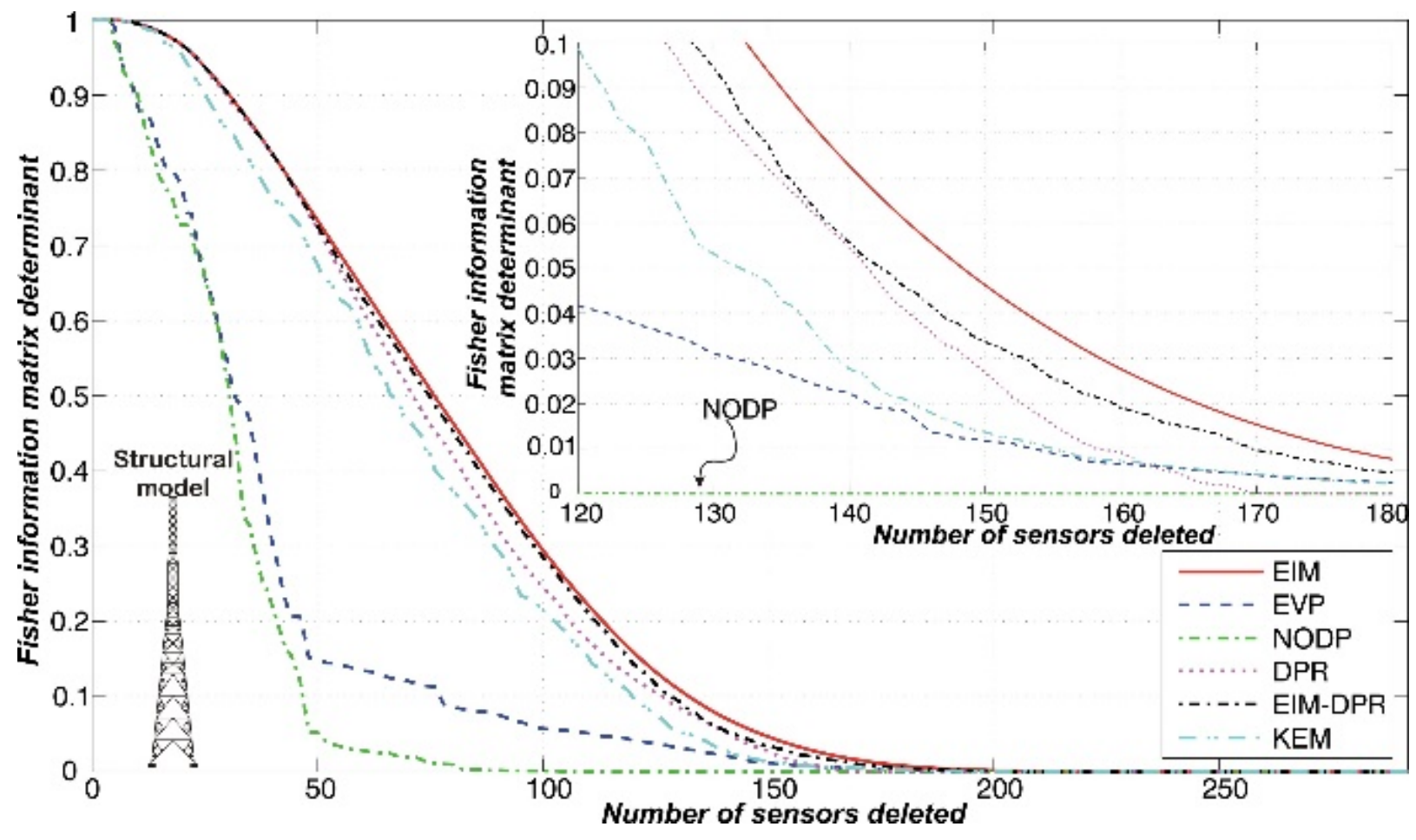

Figure 3. Fisher information matrix determinant for Tower A

One can observe that EVP and NODP select the sensors placements in reduced zones of the tower and the NODP selects a great number of vertical DOF to monitor. The most degenerate configurations is obtained by means of DPR: in this case, horizontal DOF are progressively selected from the top to the bottom of the tower. By combining the EIM and DPR, some of the sensor on the lower part of the tower (selected by means of the EIM) are removed and "placed" on the top. The most regular configurations are obtained by using the EIM and the KEM: final configurations leads to a quasi-uniform and quasisymmetric geometry of the arrays. Moreover, it is possible to observe that sensors arrays obtained by means of the EIM and the KEM are quite similar.

A comparative analysis for studying the difference between the obtained sensors arrays is based on the strength of the acquired signals: in fact, to reduce the influence of the noise, it should be as high as possible.

Therefore, to evaluate how the network topologies withstand the presence of disturbs, the Fisher information matrix determinant is a useful and efficient performance index.

In Figure 3, the Fisher information matrix determinant dimensionless value is plotted against the progressive number of the dropped candidate sensors for Tower A. It is possible to observe that the worst configurations are obtained with the NODP and the EVP. The EIM and EIM-DPF behave very well in comparison to the others criteria for a wide number of deleted sensors.
Intermediary performances are achieved by using KEM.

However, it should be remarked that these curves change their relative positions when the number of removed sensors increases (see the zoom on the corner).

\section{Tower B}

The finite element 2D model of Tower B is depicted in Figure 4. The FE model has 90 joints and two nodes connected to the base (total number of DOF is 176).

It is constituted of 14 different type frames with area in the range $691 \mathrm{~mm}^{2} \div 15280 \mathrm{~mm}^{2}$. In Figure 5, it is possible to observe that topological configurations are quite similar to the previous lattice tower: also in this case, the EIM and KEM appear to give a quite uniform distributions of the sensors along the height of the antenna. In the same way, the others configurations have some criticalities.

With reference to the Fisher information matrix determinant (see Figure 6), the optimum criteria based on the NODP provides the most sensible configuration against the noise. On the other hand, the EIM remains the best optimum criterion. 

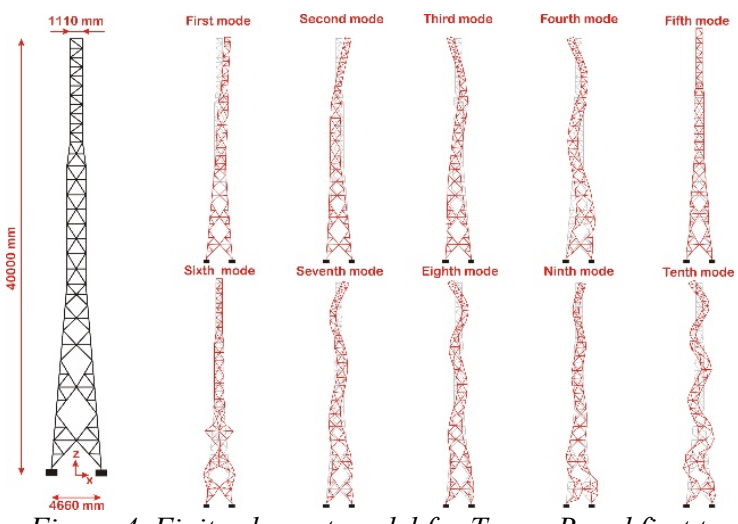

Figure 4. Finite element model for Tower $B$ and first ten mode shapes
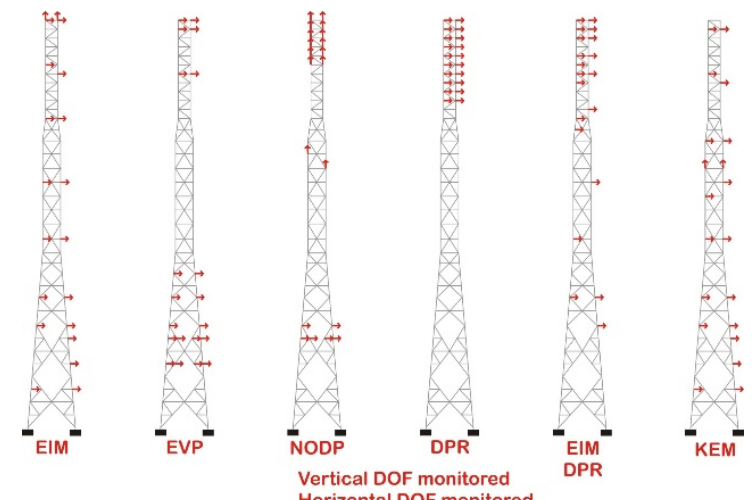

Figure 5. Sensors configurations for Tower B
Therefore, general guidelines from these benchmark cases of study can be extrapolated. Optimum sensor arrays obtained by using EIM and KEM are suitable in these structures for two reasons.

Firstly, they approximately lead to fairly uniform and symmetric sensors configurations, so that a greater capability to capture the dynamic response of the structures (especially in the modal operational analysis) is attended. Secondarily, the Fisher information matrix determinant indicates a good behaviour against the noise. With reference to the horizontal DOF, one can observe that both criteria give final configurations not excessively dissimilar. Diversely, with reference to the vertical DOFs, it is interesting to note that EIM always selects the nodes on the top while KEM chooses the nodes on the upper part of the towers but not in the same positions. This result can be analyzed with reference to the nodal mass distribution: in fact, KEM privileges nodes with greater mass, in virtue of their greater kinetic energy.

These considerations and these results could be a valid technical support to decide number and locations of the sensors. Firstly, the designer chooses a compromise between sampling frequency and number of sensors to install, taking into account the unavoidable economical and technological constraints. Subsequently, it is always advisable to perform the EIM or the KEM to define the optimum placements for the sensors and to evaluate the expected covariance matrix of the coefficient response vector by means of (3). The standard deviation $\sigma$ of the

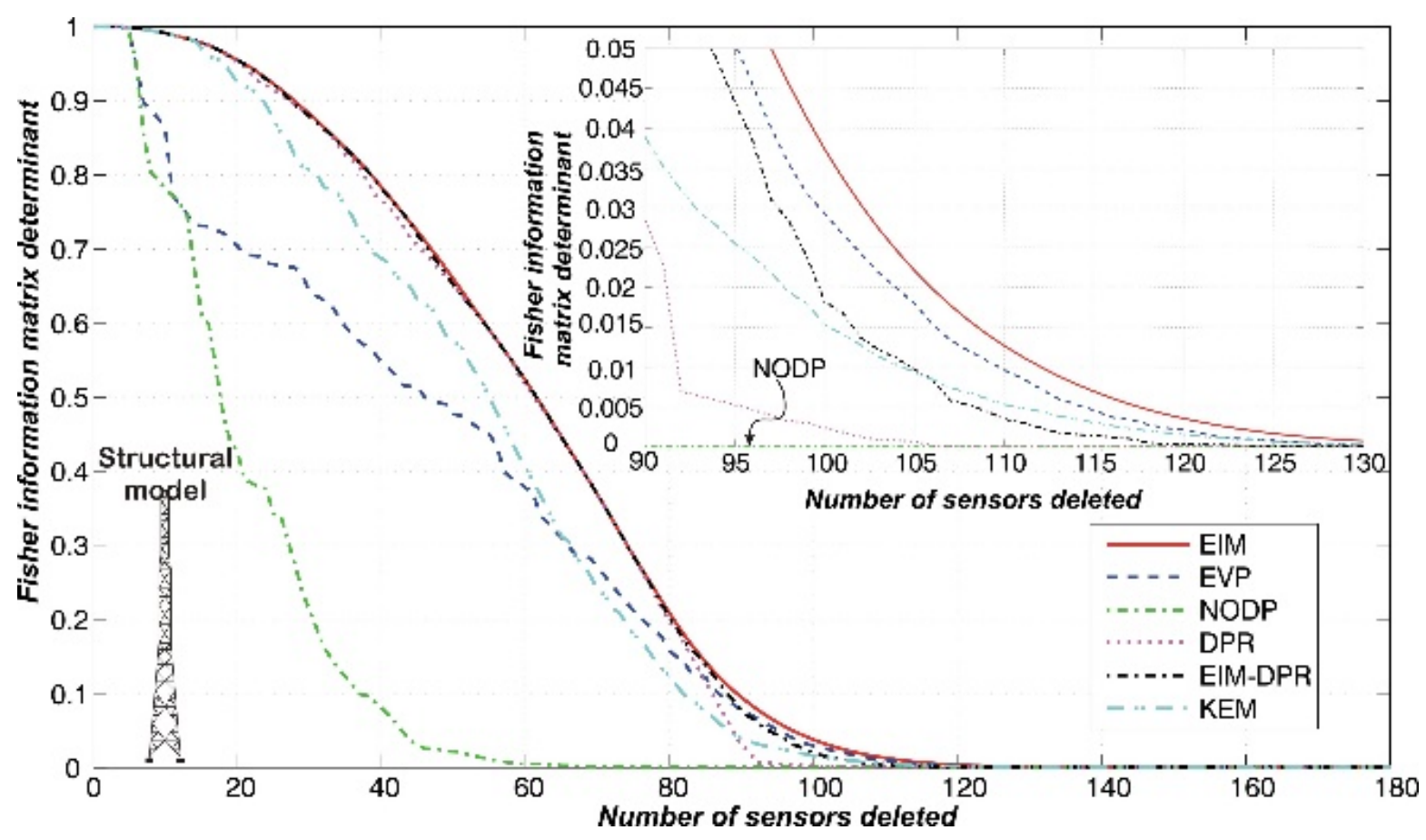

Figure 6. Fisher information matrix determinant for Tower B 
WGN can be found in the sensors data sheets or from preliminary laboratory tests.

However, in evaluating the economic efforts, it should be kept in mind that not only the number of sensors define the total cost (positions and others characteristics of the networks can sensibly modify this value).

\section{CONCLUSIONS}

The optimum design of the sensors arrays in lattice towers is addressed in the paper. Within the framework of the structural identification problems, six different optimum criteria are selected to calculate six configurations for the sensors arrays. The applications regarding two broadcasting antenna demonstrate that each optimality criteria leads to configuration very different in comparison to the others.

From a topological standpoint, the application of the EIM and KEM provide very regular arrays, so that we can expect a better performance in modal identification problems. On the other hand, the EVP, NOODF, DPR and EIM-DPR conduct to critical configurations. In order to appreciate the robustness against the noise, the performance of the calculated sensors configurations are evaluated with reference to the Fisher information matrix determinant.

In both towers, we observe that the EIM is more robust than the others optimality criteria for a wide range of sensors deleted. From our study, the EIM is the most appropriate approach for sensors placement in lattice towers, while the performance of the KEM are sufficiently good and then acceptable for this goal. The other optimality criteria appears not appropriate for sensors placement in lattice towers.

\section{REFERENCES}

Doebling, S.W., 1995. Measurement of structural flexibility matrices for experiments with incomplete reciprocity, $\mathrm{PhD}$ dissertation, Colorado University.

Gawronski W., 1997. Actuator and sensor placement for structural testing and control, Journal of Sound and Vibration, 208(1), 101-109.

Guo H.Y., Zhang L., Zhang L.L., Zhou J.X., 2004. Optimal placement of sensors for structural health monitoring using improved genetic algorithms, Smart Materias and Structures, 13(3), 528-534.

Kammer, D.C., Brillhart R.D., 1996. Optimal sensor placement for modal identification using systemrealization methods, Journal of Guidance, Control and Dynamics, 19(3), 729-731.

Liu W., Gao W.C., Sun Y., Xu M.J., 2008. Optimal sensor placement for spatial lattice structure based on genetic algorithms, Journal of Sound and Vibration, 317(1-2), 175-189.

Meo M., Zumpano G., 2005. On the optimal sensor placement techniques for a bridge structure, Engineering Structures, 27(10), 1488-1497.

Overton G., Worden K., 2004. Sensor Optimisation Using an Ant Colony Metaphor, Strain, 40(2), 5965.

Papadimitriou C., 2004. Optimal sensor placement methodology for parametric identification of structural systems, Journal of Sound and Vibration, 278(4-5), 923-947.

Shi Z.Y., Law S.S., Zhang L.M., 2000. Optimum Sensor Placement for Structural Damage Detection, Journal of Engineering Mechanics, 126(11), 1173-1179.

Shih Y.T., Lee A.C., Chen J.H., 1998. Sensor and actuator placement for modal identification, Mechanical Systems and Signal Processing, 12(5), 641-659.

Wang M.L., Heoz G., Satpathiy D., 1998. A health monitoring system for large structural systems, Smart Materias and Structures, 7(5), 606-616.

Worden K., Burrows A.P., 2001. Optimal sensor placement for fault detection, Engineering Structures, 23(8), 885-901. 